\title{
Effect of the pressed ceramic filters on the reoxidation process
}

\author{
Marek Brůna ${ }^{1, *}$, Dana Bolibruchová $^{1}$, Richard Pastirčák ${ }^{1}$ \\ ${ }^{1}$ University of Žilina, Faculty of Engineering, Department of technological engineering, Univerzitná \\ 1, 01026 Žilina, Slovakia
}

\begin{abstract}
Article is focused on reoxidation processes during filtration of aluminium alloys. Many of our experiments proven, that when using filtration media in gating system, reoxidation occurs. Main goal of this article is to point out reoxidation problems occurring during filtration of aluminium alloys. Reason to conduct this work was anomalies discovered by experimental casts, which pointed out that after filtration of primary alloys, small oxide films was detected. Oxides was absent, when we poured without filtration media. Simulation software ProCAST was used during our research.
\end{abstract}

Keywords: Filtration, Aluminium alloys, simulation software

\section{Introduction}

Oxygen solubility in aluminum is very low, less than one oxygen atom in $10^{35}$ to $10^{40}$ aluminum atoms, yet it is generally known that aluminum and its alloys are full of oxides. However, oxides cannot penetrate the inner layers of the melt by chemical reaction with oxygen. Oxygen reacts only with the surface layer of the melt and the penetration of the oxides into the inner layers can occur only through the mechanical processes during which the surface oxides are entrained into the internal volume of the melt. Oxide films can be formed in aluminum alloys in 0.01 seconds and already have a thickness of $1 \mu \mathrm{m}$ after 1 second, after 10 seconds they are thick about $10 \mu \mathrm{m}$. If the melt surface is exposed longer time to an atmosphere in the mold cavity, the resulting oxide film will be thickening during all filling process. However, if turbulence occurs, this thick oxide film will furl and entrain into the inner volume of the melt (future solidified casting), or it may cause melt-in-entry problems from wider cross-sections to narrower.

Oxide films can be considered relatively harmless when found on the surface of the melt. Solid oxide film grows from the melt surface, as each metal atom is combined with the incoming gas surrounding atoms. [1]

As a result of turbulence, the surface oxide film is entrained (fig. 1) and furl in a compact shape, resulting in a change in size. The oxide film changes the shape from a 1 $\mathrm{mm}$ to $10 \mathrm{~mm}$ sheet on a substantially smaller, furled area of 0.1 to $1 \mathrm{~mm}$. In this compact

\footnotetext{
* Corresponding author: rapture.marko@gmail.com

Reviewers: Eva Tillová, Mariana Pajtášová
} 
form, most of the oxide films are able even to penetrate through the pores of the filter media placed in the gating system.

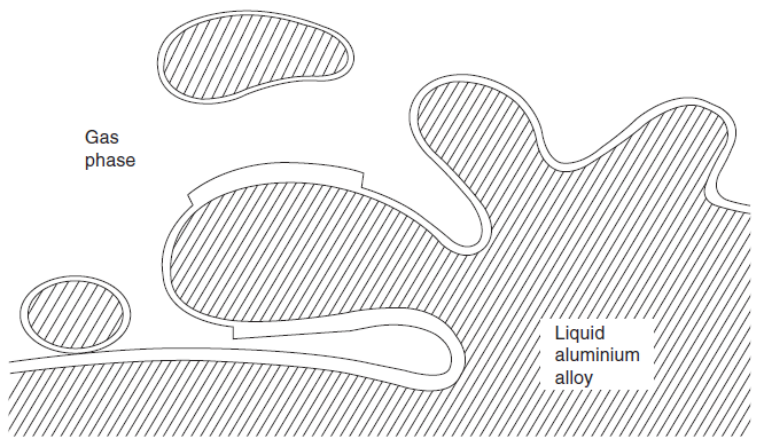

Fig. 1. Scheme of a surface entrainment event [1]

At the moment, when it comes to filling the mold, oxide films find themselves in a new, relatively quiet environment. Movement occurs only due to the heat transfer and slight motions of the liquid metal where the Reynolds number reaches only low values. The new environment around the oxide films creates the conditions for its gradual development, opening and rapid growth up to ten times its original size. In the developed form, the oxide film has the ability to substantially weaken the cross section of the casting and thereby affect its resulting mechanical properties. The driving force for redeveloping of the oxide film may be a number of mechanisms (most often by precipitation of hydrogen between the layers of the oxide film) $[1,2]$.

This does not mean, however, that the use of filters in the casting of aluminum alloys is not justified. The positive effect of the filters lies mainly on the fact that the narrow channels in the filters support the laminar flow pattern.

The aim of the present paper is to investigate the effect of the pressed ceramic filter on the reoxidation of aluminum alloy $\mathrm{AlSi} 7 \mathrm{Mg} 0.3$ by simulating the flow in the area of the filtration medium [3].

\section{Experimental part}

Aluminum alloy AlSi7Mg0.3 casted into a metal mold with ceramic pressed filters of three different thicknesses (fig. $2.50 \times 50 \times 10-15-22 \mathrm{~mm}, 314$ channels, channels diameter 2, $2^{ \pm 0,15} \mathrm{~mm}$ ) in gating system was used for experimental work. Pouring temperature was $730 \pm 5^{\circ} \mathrm{C}$. Placement of the filtration media and scheme of the whole experimental apparatus is shown in fig. 3 .

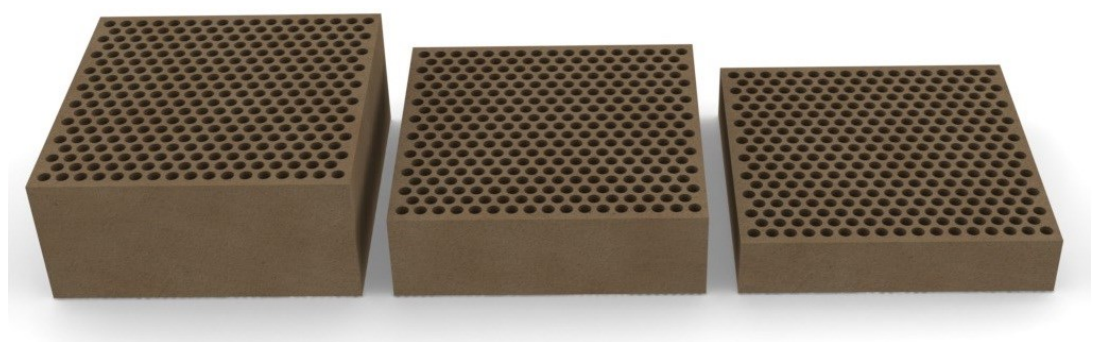

Fig. 2. Ceramics pressed filters $(22 \mathrm{~mm}-15 \mathrm{~mm}-10 \mathrm{~mm})$ 


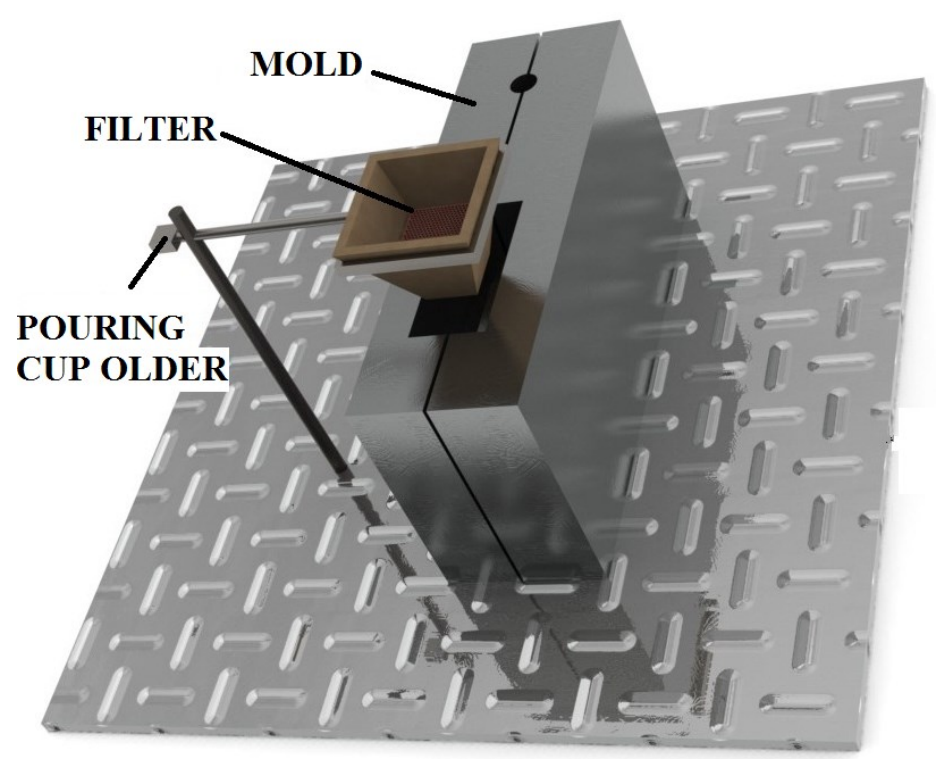

Fig. 3. Measurement apparatus

In order to observe the nature of the flow in the near vicinity of the ceramic filter, ProCAST software was used to simulate the filling of the mold. Instead of the standard function for the analysis of the melt flow in the filter area offered by the software, an innovative approach to the problem was proposed. Individual filter media were imported into the simulation software with precise geometry (created in the Solidworks CAD program). This fact has a considerable impact on the complexity of the simulation process, especially on the surface and subsequently the volume mesh creation, which eventually resulted in the time required for simulation (in the case of a pressed filter up to approximately 350 hours of calculations). However, this mode of simulation has great advantages over the conventional method offered by the software. By using normal method, the software does not take into account the geometry of the individual channels. The main role of the simulation method used is the possibility of more accurate observation of the melt flow in the area of the filtration medium. During the experiments, we mainly focused on the nature of the melt flow before the filter and behind the filter, in terms of the formation of the reoxidation products. The assumption is that the filter itself presents an obstacle in the gating system which may cause a turbulent flow by its presence on the inflow side of the filter. Another important and observed factor is the phenomenon behind the filter, where the continuous melt flow is divided on the sum of small flows from the individual filter channels which may result in a considerable increase of the free area exposed to the atmosphere.

Figure $4 \mathrm{a}$ shows a mold cavity and filter with thickness of $10 \mathrm{~mm}$ before the pouring, Fig. $4 \mathrm{~b}$ represents the start of the mold filling and the first contact of the melt with the filter. Purple color of the melt is the liquid metal with the lowest probability of reoxidation occurrence (created during mold filling, because simulation software does not provide information about oxides incurred in previous metallurgical stages) and shades of blue, green, yellow and red, the possibility of oxide occurrence increases. Whole color spectrum in all figures represent interval from 0 to $1 \mathrm{~cm}^{2} / \mathrm{sec}$, that means area of free surface possibly covered in oxide in one second. 
At the inlet side of the filter in the initial stage of filling, the melt does not accumulate and all its volume flows through the filter filling the mold cavity in a smooth and continuous flow of liquid metal, which is to counteract the formation of oxides optimal conditions. During the accumulation of the melt at the inlet side there is a prolonged contact of the melt free surface with the atmosphere, which would lead to increased formation of oxide films. Continuous stream of liquid metal on the downstream side may also be seen as a positive phenomenon, the separation of the stream to a smaller streams could increase the melt surface exposed to the atmosphere $[4,5]$.

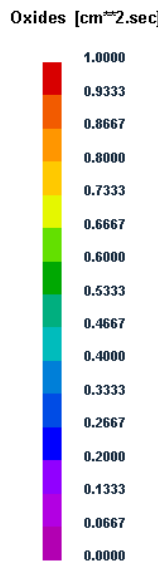

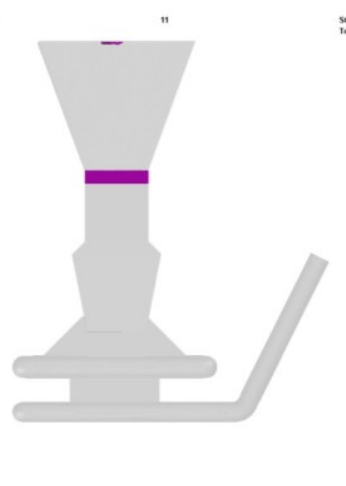

a)

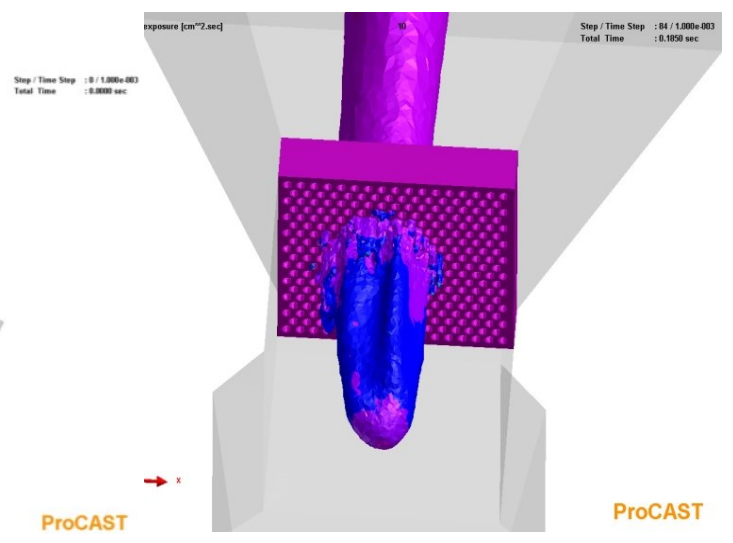

b)

Fig. 4. Simulation of starting stage of filling -filter thickness $10 \mathrm{~mm}$

Comparing Figures $5 \mathrm{a}$ and $5 \mathrm{~b}$, which represent the later stages of filling, it can be stated that most oxide contamination occurs when the melt is on the bottom of the mold (red melt color on the mold bottom). The critical free-fall velocity of aluminum melt according to [3] is $0.5 \mathrm{~m} / \mathrm{s}$. The author claims that, once the given value has been exceeded, turbulence cause to submerge surface oxides into the internal volume of the melt and new oxide films will form on the surface of the melt. The simulation software has determined that the melt will have velocity up to $2.2 \mathrm{~m} / \mathrm{s}$ when it reaches the bottom of the mold. Negative phenomena is also the reflection waves originating in the central area of the mold, which are also shown in red.

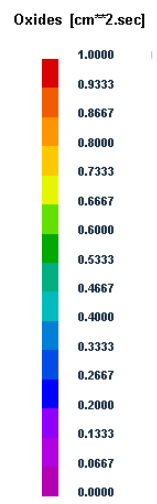

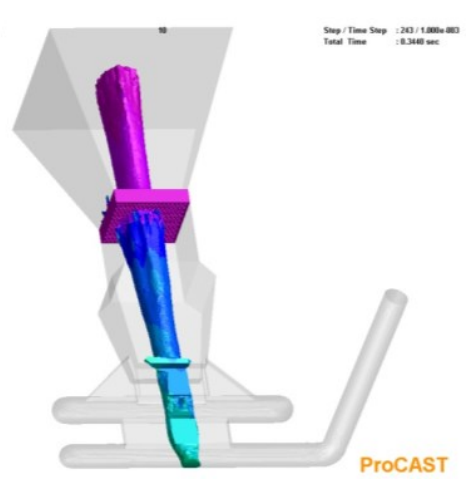

a)

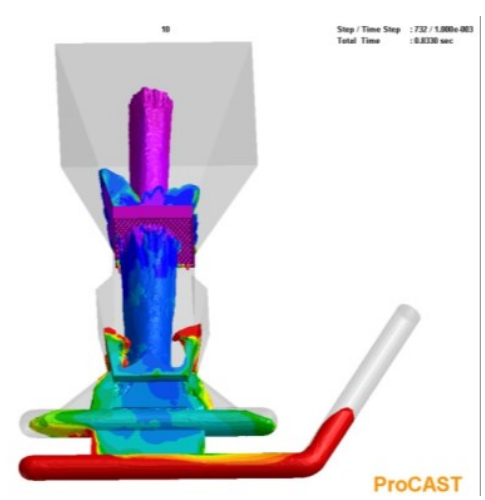

b)

Fig. 5. Simulation of later stage of filling-filter thickness $10 \mathrm{~mm}$ 
Fig. 6a shows a mold cavity and filter with thickness of $15 \mathrm{~mm}$ before the pouring. In fig. $6 \mathrm{~b}$ can be seen that melt passage through a thickened filter press produces the formation of separate melt streams emerging from the individual channels of the filter. This negative effect was reflected in an increase in the area of the melt exposed to the atmosphere in the inlet system, as can be seen in fig. 7 (melt color yellow to orange on the filter side) [6].

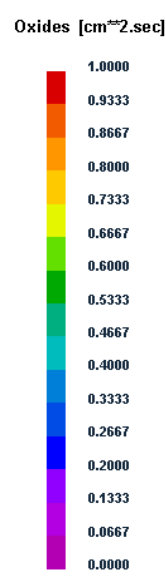

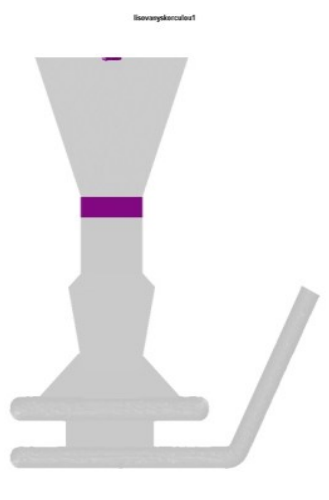

a)

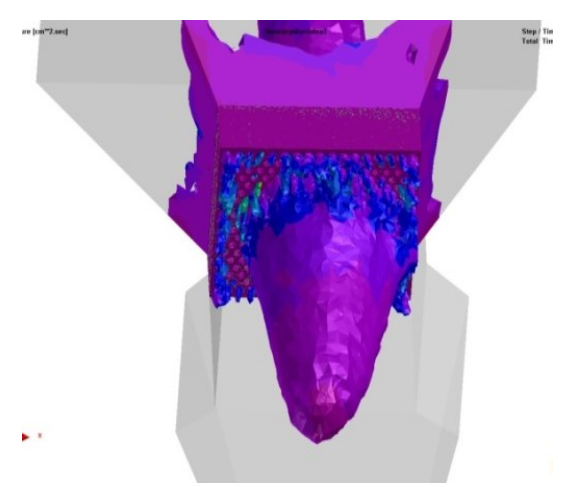

b)

Fig. 6. Simulation of starting stage of filling -filter thickness $15 \mathrm{~mm}$

In Figure 7, redundant amount of melt at the inlet side of the filter can be seen; the red color at the free surface indicates the formation of oxides in the region, consequently they will be pulled deeper into the mold cavity. The excessive amount of melt at the inlet side of the filter and the turbulent flow pattern in this zone allows the oxide films to furl into compact form and thereby penetrate through the filter despite all filtration mechanisms. Due to the greater thickness of the filter used, the impact velocity of the melt was reduced to the bottom of the mold $-1.7 \mathrm{~m} / \mathrm{s}$ (the color of the melt on the bottom of the mold blue and green).

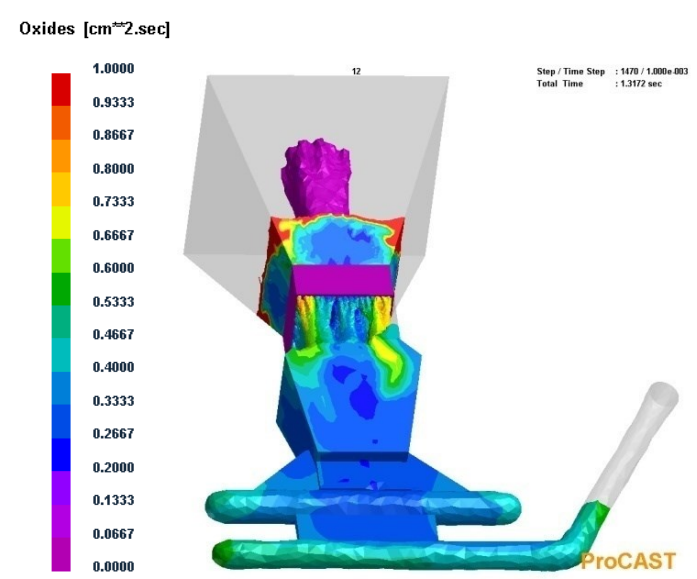

Fig. 7. Simulation of later stage of filling -filter thickness $15 \mathrm{~mm}$

Fig. 8a shows a mold cavity and filter with thickness of $22 \mathrm{~mm}$ before the pouring. On fig. $8 \mathrm{~b}$, it is possible to notice the formation of an oxide film layer lining the entire volume of the melt at the inlet side of the filter. The impact velocity of the molten metal on the 
mold bottom at a $22 \mathrm{~mm}$ filter thickness was $1.3 \mathrm{~m} / \mathrm{s}$ (the color of the blue and purple mold bottom - Figure 9).
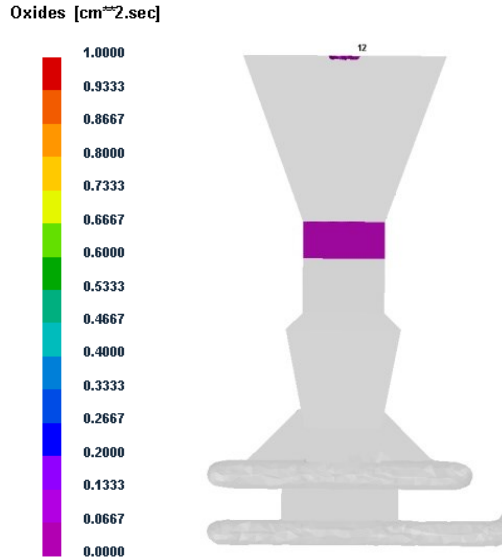

a)

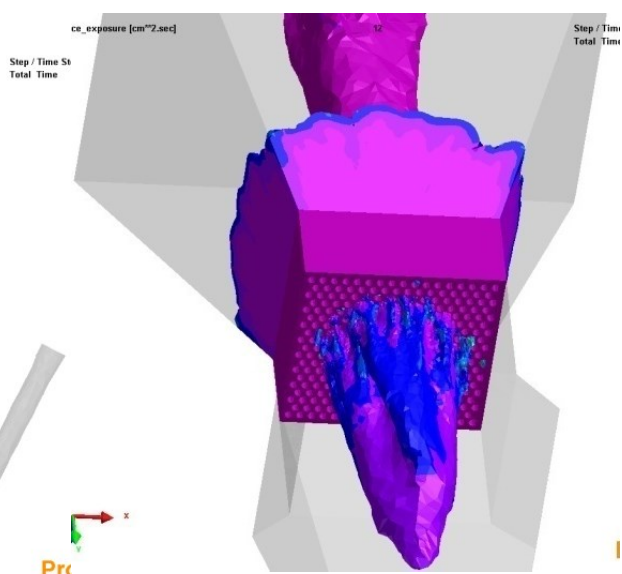

b)

Fig. 8. Simulation of starting stage of filling -filter thickness $22 \mathrm{~mm}$

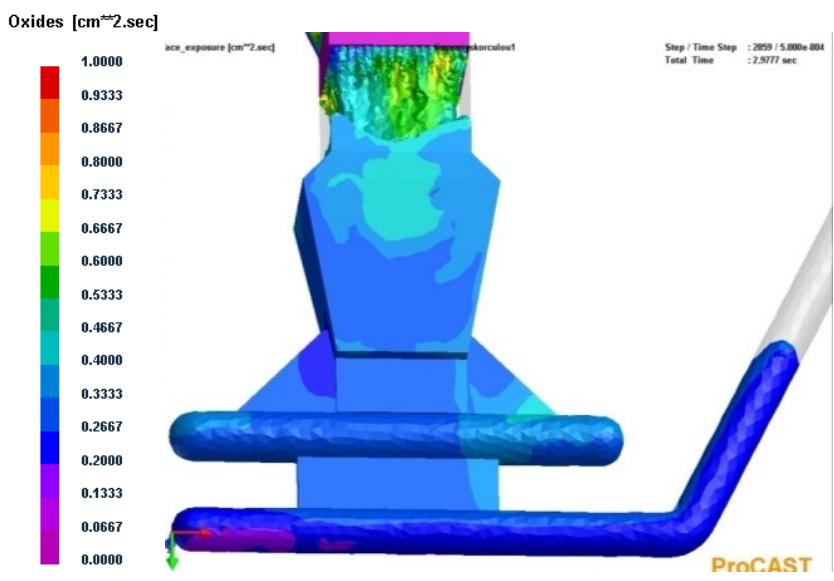

Fig. 9. Simulation of later stage of filling -filter thickness $22 \mathrm{~mm}$

Figure 10 shows a comparison of the filling of the metal mold in the area of the flow of the filter medium (Figure 10a - Thickness filter $10 \mathrm{~mm}$, Figure 10b - $22 \mathrm{~mm}$ thickness filter). In order to have a comparison value, in both cases the simulation was stopped at the same timeout $(0.22 \mathrm{~s})$. Seeing that with a different thickness of the filter, there is also a different way of filling the mold. In the case of a thinner filter, filling occurs faster, the melt does not stick to the inlet side of the filter, there is no excessive turbulence in the area, but the melt reaches higher speeds. 

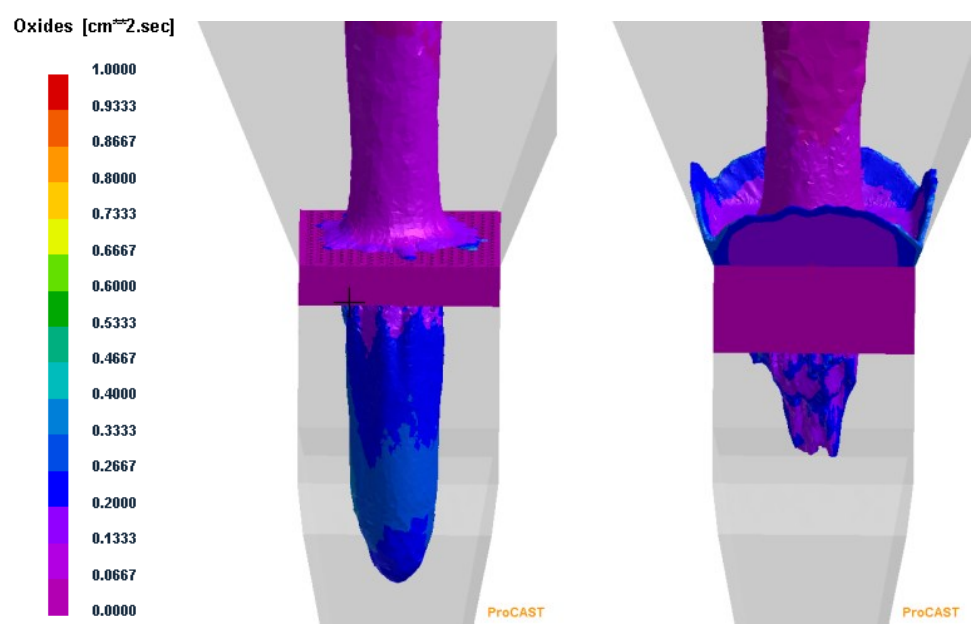

Fig. 10. Flow comparison -filter thickness $15 \mathrm{~mm}$ (left), $22 \mathrm{~mm}$ (right)

\section{Conclusion}

The aim of this work was to point out an atypical view of the mechanism of ceramic pressed filter effect during the pouring of aluminum alloys. The results of the work paradoxically point to increased reoxidation processes compared to the refining effect during filtration of aluminum alloys and extend the set of works that deal with the simulation of the liquid aluminium flow. The aim of the thesis was not to determine the optimum location of the filter in the inlet system, but to visualize the changes in the filling process, taking into account the change of the used filter thickness. Using a detailed analysis of the simulations of all three variants, it can be concluded that the thickness of the used filter has a significant effect on the flow of melt flow, melt velocity and the amount of oxide generated in the gating system. When using a $10 \mathrm{~mm}$ thick filter, the excessive flow of the melt is not accumulated at the inlet side, but the flow rate on the drain side is considerably larger than the thicker filters. The simulation software estimated that the optimum filter thickness of $22 \mathrm{~mm}$ is optimized for the purpose of suppressing the excessive reoxidation since the melt flow rate at the bottom of the mold approximates to a subcritical value of $0.5 \mathrm{~m} / \mathrm{s}$, and the long filter channels more strongly contribute to the laminarization of the melt stream.

This work was created within the framework of the VEGA project grant no. 1/0494/17 with the title: Vplyv pretavovania recyklovaných hliníkových zliatin na úžitkové vlastnosti náročných odliatkov automobilového priemyslu.

\section{References}

1. J. Campbell, Complete casting handbook second edition, Butterwirth-Heinemann (2015)

2. L. Zhang, L. Damoah, S. Li, W. Abebe, Mechanisms of Inclusion Removal from Aluminum through Filtration. Light Metals, TMS, 649-655 (2008)

3. J. Campbell, Casting practice - the 10 rules of castings. (Oxford, ButterworthHeinemann, 224 p., ISBN 07506-4791-4, 2010) 
4. M. Tropp, M. Handrik, P. Kopas, M. Sága, Computer simulation of induction bending process. Pipeline \& Gas Journal, ISSN 32-0188, (2012)

5. R. Raiszadeh and W. D. Griffiths, The Effect of Holding Liquid Aluminum Alloys on Oxide Film Content. Metallurgical and Materials Transaction 42B, 133-143 (2011)

6. D. Bolibruchová, Filtrácia hliníkových zliatin. (Žilina, Žilinská univerzita, 203 p., ISBN 978-80-55-0342-7, $1^{\text {st }}$ ed., 2011) 